\title{
Molecular Engineering of the Geobacillus stearothermophilus a-Amylase and Cel5E from Chlostridium thermocellim; In Silico Approach
}

\author{
Ibrahim Torktaz ${ }^{1,2}$, Jafar Hemmat ${ }^{* 2}$, Ali Asghar Karkhane ${ }^{\dagger 1}$, Garshasb Rigi ${ }^{3}$, Amin Rostami ${ }^{4}$, Jafar \\ Khezri $^{1}$, Reza Behroozi ${ }^{1}$ \\ ${ }^{1}$ National Institute of Genetic Engineering and Biotechnology, Tehran, Iran \\ ${ }^{2}$ Biotechnology Department. Iranian Research Organization for Science and Technology (IROST) Tehran-Iran. \\ ${ }^{3}$ Department of Genetics, Faculty of Basic Science, University of Shahrekord, Shahrekord, Iran \\ ${ }^{4}$ Department of Basic Sciences, Faculty of Paramedical Sciences, Shahid Beheshti University of Medical Sciences, Tehran, Iran
}

"Corresponding author: Jafar Hemmat, Biotechnology Department. Iranian Research Organization for Science and Technology (IROST) Tehran-Iran. Telefax: +98 21 56276636, E-mail: J.hemmat@irost.org

${ }^{\dagger}$ Co-Corresponding author: Ali Asghar karkhane (karkhane@nigeb.ac.ir)

Received: 6 Jul. 2015; $\quad$ Revised: 24 Oct. 2017; $\quad$ Accepted: 25 Oct. 2017; $\quad$ Published online: 11 Aug. 2018

\begin{abstract}
Background: Considering natural thermal stability, Geobacillus stearothermophilus amylase and Cel5E from Clostridium thermocellum are good candidates for industrial applications. To be compatible with the industrial applications, this enzyme should be stable in the high temperatures, so any improvement in their thermal stability is valuable.

Objectives: Using in silico approach and identifying point mutations in the structure amylase of G. stearothermophilus and Cel5E from C. termocellum we tried to increase thermal stability of the enzymes along with their catalytic activity to reach a new industrial amylase with higher thermostability and an improved function.

Materials and Methods: In this study we predicted the 3D structure of the enzymes, then simulated the molecular docking study using MolDock, PLANTS, and Lamarkian genetic algorithm as scoring functions for the docking and in silico engineering of the protein aiming to increase the thermal stability and catalytic activity.

Results: A series of thermal stability increasing point mutations were exerted around the active site of the enzyme, then by docking procedure, the binding affinity was measured and finally a list of mutations which theoretically improved the increased thermal stability as well as catalytic activity were proposed.

Conclusions: Based on the in silico results obtained the modified enzymes seems to be suitable candidates for considering in both laboratory and industrial scales.

Keywords: Amylase; Catalytic activity; Cel5E; Geobacillus stearothermophilus; Industry; Thermal stability
\end{abstract}

\section{Background}

Over the past decade, a number of industries have met with an increasing demand for the thermostable enzymes in order to catalyze different processes such as biodegradation and biotransformation $(1,2)$, knowing the fact that in many cases, by increasing the temperature of the processing, the rate of the reaction will also increase. On the other hand, an increased thermostability of the enzyme would result to a longer storage time with an optimal catalytic temperature, a lower vulnerability to the organic solvents, a lower risk of contamination, and activity loss during pre- processing of the raw materials during the industrial procedure (3-5). Among the various industrially used thermostable enzymes, amylases have drawn the greatest interest.

$\alpha$-amylase family (an endo-acting 1,4- $\alpha$-Dglucanglucanohydrolase) are the main group of amylolytic enzymes (6) which are involved in the processing of the oligosaccharides and polysaccharides. The importance of the amylases lies in their ability to hydrolyze starch (a polymer of glucose with glycosidic bond that exist in the forms of amylose and amylopectin), glycogen, and other related saccharides into the lower

Copyright (C 2017 The Author(s); Published by National Institute of Genetic Engineering and Biotechnology. This is an open access article, distributed under the terms of the Creative Commons Attribution-NonCommercial 4.0 International License (http://creativecommons.org/licenses/ by-nc/4.0/) which permits others to copy and redistribute material just in noncommercial usages, provided the original work is properly cited. 
molecular weight products such as glucose as maltose units $(7,8)$. It was shown that the presence of the two aspartic acid residues (Asp) and one glutamic acid (Glu) residue are essential for the catalytic activity of the $\alpha$-amylase. These residues are conserved among different species (9). $\alpha$-amylases from different sources seem to have a similar three-dimensional structure with three domains: domain $\mathrm{A}$, that has a central $(\beta / \alpha) 8$ barrel, domain B, and domain $\mathrm{C}$ entailing a Greek key motif (9). Due to its utilities in the industries including fuel alcohol production, detergent industry, food, textile, paper, and pharmaceutics, it is estimated that this class of enzymes accounts for more than $25 \%$ of the world's enzymes market (8). Starch hydrolysis is performed at a temperatures over $100{ }^{\circ} \mathrm{C}$. This highlights the importance of the thermotolerant amylolytic enzymes to remain stable at such a high temperatures (10). As well, it emphasises the importance of developing enzymes with the improved functional properties.

Since thermophilic organisms naturally live in the harsh conditions, many thermostable enzymes have been isolated from these organisms for the first time. As a hyper thermophile bacterium, G. stearothermophilus, a Gram-positive endospore-forming bacterium grows at the temperatures above $70^{\circ} \mathrm{C}$ and distributes in soil and hot springs (11). This bacterium produces a number of hyper thermophile enzymes among which the amylase is an important industrial enzyme. It is reported that the half-life of the amylase produced by this species is 50 minutes at $90{ }^{\circ} \mathrm{C}$ and $\mathrm{pH} 6.5$ (12). Moreover, C. Thermocellum is a well known thermophilic bacteria. Interestingly, not all thermostable enzymes were isolated from thermophile bacteria. While it is not a thermophilic bacterium, Bacillus licheniformis's amylase (BLA) like Cel5E from Clostridium thermocellum provides a good example of a thermostable enzyme which remains active for several hours at high temperatures. Bacillus licheniformis's amylase (BLA) is an enzyme with 483 amino acids length and a half-life of 270 minutes at $90{ }^{\circ} \mathrm{C}$ and $\mathrm{pH}$ 6.5. It was shown that there is a high similarity between the amino acid sequence of the BLA with that produced by B. Stearothermophilus. Although several attempts were done in the past in order to improve the thermostability of the amylase produced by $B$. Stearothermophilus (13), however, BLA was subject of more attemps in this regard (12). Due to its high thermostablity, BLA is intensively used by protein engineers as a model for elucidating the molecular mechanisms, rendering high thermostability capacity to the enzyme, as well as widely application as an industrial enzyme. Comparing the structures and sequences of the the enzymes with their thermostable counterparts can provide valuable clues for engineering proteins in order to gain more thermal tolerable and improved enzymes especially for industrial applications.

In fact, the thermostability of an enzyme determines how long it remains active in the defined temperature (i.e. the more thermostability is equal to a more prolongeed enzyme activity). Thermostability of an enzymes can be influenced by Van der Waals interactions, hydrophobicity, hydrogen bonds, ion pairs, and non-covalent interactions (14). Many different methods have been proposed for the prediction of thermostability changes in the proteins based on protein mutation (15) including measuring and comparing the free energy changes before and after exerting the mutation (16). The alteration in the free energy of denaturation $(\Delta \mathrm{G})$ or $\Delta \Delta \mathrm{G}$ is a well-known indicator for the protein thermostability changes (17). These alterations in $\Delta \mathrm{G}$ can be made by exerting a point mutation in certain residues of the protein sequence and further evaluation of its $\Delta \Delta \mathrm{G}$ to find any refinement out in its thermostability (18).

\section{Objectives}

In the present study, in silico 3D structure prediction method based on Hidden Markove Model (HMM) algorithm was used. Using in silico approach and identification of the point mutations in the structure of amylase from G. stearothermophilus and Cel5E from $C$. thermocellum, we tried to increase the thermal stability of the enzymes along with their catalytic activity to reach new industrial amylases with a higher thermostability and improved function. Since G. stearothermophilus amylase is not enough thermostable (i.e., it is rapidly deactivated at the high temperatures), it cannot be an industrially usable enzyme. For increasing the catalytic activity, we used an in silico approach to a simulated molecular docking study using MolDock and PLANTS as a scoring function for docking. The results were also compaired by single point mutations within the structure of Cel5E from Clostridium thermocellum.

\section{Materials and Methods}

\subsection{Protein Model Prediction and Structural Assessment}

Hidden Markov Model (HMM) algorithm provided by the SAM-T08 web server was used for protein modeling of the $\alpha$-amylase (19). Briefly, a sequence of amino acid in FASTA format was extracted from NCBI (Accession No: YP_003252634) and introduced to the SAM-T08 server as a query. After providing Multiple Sequence alignments (MSAs), MSAs were imputed to 
an artificial neural network in order to predict different local structural properties. Next, HMMS were built from MSAs using the sequence alignment and modeling (SAM) tool site and predicted local structures. Then, the resulting HMMs were used to search PDB structures for the potential templates for the structure prediction. In the case of Cel5E, the PDB template of 4U3A was used as the template. In order to evaluate the predicted model, Q-mean score was used (20). The Q-mean is a hybrid scoring function, depicting the main geometrical features of the protein structures, ranging from 0 to 1 . Although scores near 1 mean that the model is similar to the crystallographic structure. Also, prosa Z score was used to reevaluate the accuracy of the predicted model.

\subsection{Thermal Stability Prediction}

For thermal stability prediction, PoPMusic algorithm was used (21). The predicted structure was used as the input for the algorithm. The PoPMusic was used to mutate each amino acid into the 19 other amino acids and after each mutation it was evaluated for the subsequent changes in the folding free energy. Calculating the folding free energy determines the stability changes caused by the single-site mutations.

\subsection{Molecular Docking Study}

Molecular docking was performed using Molegro Virtual Docker v 5.5 (MVD) software (22) using Protein-Ligand ANT System (PLANTS) scoring function. In order to re-evaluate the docking results and increasing the accuracy of computation, MolDock score was calculated. For both native and mutated protein models, docking procedure was performed in the same conditions where a grid resolution was set at $0.3 \AA$ and a maximum iteration number was set to 1500 in 10 runs for each model. The energy minimization and optimization of the hydrogen bonds were determined to be performed after the docking operation. After preparation of the molecular structures of the enzyme and ligands, the charge calculation and assignment to the models were done by the software.

As an alternative scoring function, AUTODOCK 4 software was used which was simulating the solution condition in the presence of neutralizing ions. AUTODOCK 4 shows the contact of the protein and ligands in $3 \mathrm{D}$ crystal structure and benefits from the Lamarckian genetic algorithm (LGA). For MolDock and PLANTS docking evaluations, all docking operation's radius was set at 17 and 14 to cover the entire volume of the main cavity of the amylase and Cel5E respectively. Also, the coordinate of substrate binding for all operations was set fixed to X: 77.67 , Y:
30.45, and Z: 77.63 for mylase and X: -0.91 , Y: 34.86 and Z: -5.41. for Cel5E. Docking operations were conducted under the same conditions for the native and mutated models with a grid resolution of $0.3 \AA$ and maximum iteration number of 1500 in total 10 runs for each model. Following the docking operations, energy minimization and optimization of hydrogen bonds were performed. Also in AUTODOCK software charges were calculated by AutoDock and added to the both protein and ligand structures. Both ligand and protein were treated as the flexible structures with the $6+\mathrm{rl}$ degrees of the freedom and $\mathrm{rp}$ torsional degrees of freedom for ligand and protein respectively in this stochastic search algorithm. This method minimizes the scoring function by using pheromone model and local search to assign the optimal values for each degree of freedom. More information in details is provided as the supplementary data.

\subsection{Mutation Selection}

The point mutations were selected and the desired mutations were applied in the 3D structures using Modeller v9.12 software. The PopMusic web server was used for prediction of protein stability upon point mutations. The mutant enzymes with an increased stability were selected as candidates for further mutations aiming at increasing the affinity of the substrate to the catalytic site of the enzyme.

\section{Results}

The family of $\alpha$-amylase enzymes have several advantages in the industrial applications (8). For being usefully applicable in the industry, enzymes should be stable at high temperatures. Many methods have been subject of thermostablity prediction of the proteins according to protein mutation $(15,16)$. During previous studies, enzyme thermostability was evaluated by an alteration in the free energy of denaturation using a point mutation in certain residues of the amino acid sequence $(17,18)$ and random mutageneses $(23,24)$. In this study, computer - based 3D structure prediction method based on Hidden Markove Model (HMM) algorithm was used. The results are explained and discussed in the four below sections.

\subsection{Protein Model Prediction and Mutations}

In this study, a prediction method based on sequence homology (SAM-T08) was used to gain a model for $G$. stearothermophilus $\alpha$-amylase. We used HMM due to high query-template coverage. So, the output model of SAM-T08 was totally covered the query sequence. The final model is depicted in Figure 1. This method is also 


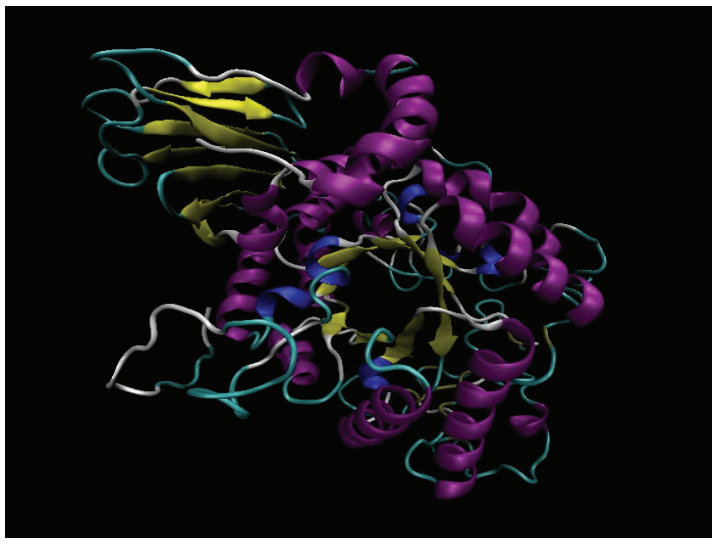

Figure 1. The structure of G. stearothermophilus amylase as predicted by HMM algorithm.

tested in the structure of Cel5E from $C$. thermocellum and compaired with its crystal structure (4U3A).

The Q-mean assay showed that the predicted structure assay of 0.69 indicates the high quality of the predicted model. Also an alternative assay was done to evaluate the accuracy of the predicted structure. The prosa Z-score determines the accuracy of the structure in $\mathrm{X}$-ray or NMR zone. The result of prosa assay is depicted in Figure 2. The obtained data have indicated that our predicted structure gained the score of 7.83 which indicates that our predicted structure has X-ray quality.

\subsection{Molecular Docking Study}

To determine which mutations theoretically increase

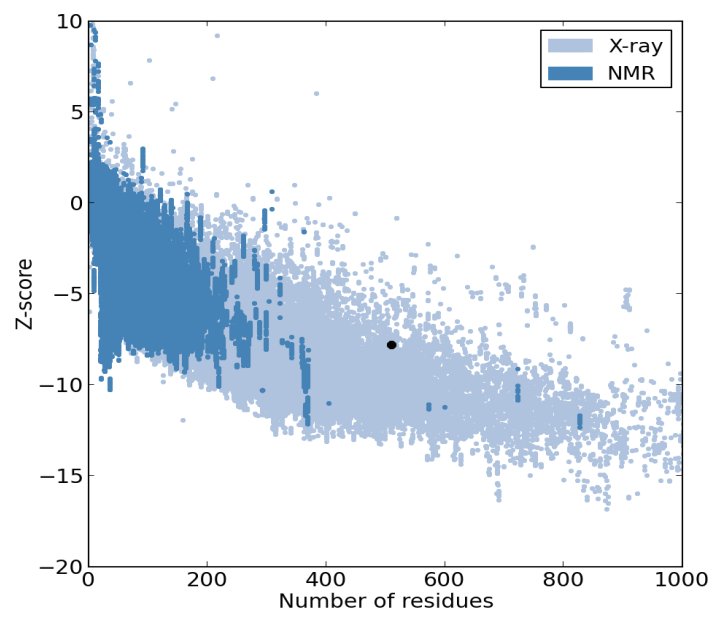

Figure 2. The prosa Z-score of G. stearothermophilus alpha amylase predicted by the modeller V 9.12 software. The score of 7.83 indicates that the predicted model has an X-ray quality. the substrate binding affinity of G. stearothermophilus, a partial polymer of amylose (substrate of amylase) was obtained from PubChem database (CID 53477771) and docked against the predicted active site of all mutant models as well as a native model. In the case of Cel5E, the ligand was extracted form PDB: 3AOF. For computer simulated docking operation, MVD and AutoDock software's with MolDock, PLANTS, and Lamarkian Genetic Algorithm as docking scoring functions were used to decrease simulation errors. The docking radius coordinate was set on the active site of the enzyme. The active site predicted as the main cavity by cavity detection algorithm of MVD.

\subsection{Mutational Analysis}

According to PopMusic output, 13 amino acid positions were selected as candidates for point mutations for amylase, including Ala 85, Met 132, Lys 133, Asp 137, Asp 210, Gly 211, Arg 213, Asp 215, Gly 211, Arg 213, Asp 215, Gly 243, and Glu 244. And Asn 207 was best candidate for Cel5E. Although these amino acids were around the active site, they were neither substrate binding nor catalytic residues. It is clear that mutation at these amino acid positions can affect the active site cavity and any minor change in the substrate binding area can lead to alteration in binding avidity. With this assumption, first mutations were performed in the 3D model by using Modeller and the topology of the mutant models were obtained. Then, the molecular docking operation was simulated to study alteration in the binding avidity as well as a change in the delta $\mathrm{G}$ of the ligand binding followed by partial changes in structure which are caused by point mutations. Table $\mathbf{1}$ describes alteration in the thermal stability as well as substrate binding upon each point mutation.

\section{Discussion}

Comparing the provided data in the Table 1 with the native amylase, there are 5 mutant types which were indicated with a higher ligand binding affinity as well as delta $G$ of the thermal stability. In position 137 the native amino acid is aspartic acid and as depicted in Figure 3 and Figure 4 it is located in the middle of a beta sheet and is surrounded by the hydrophobic amino acids. So, it is rational that replacing aspartic acid 137 with a hydrophobic amino acid leads to the strengthening of the hydrophobic core in the middle of the beta sheet. Replacing ASP 137 with isoleucine has changed the thermal stability (in delta delta G scale) -0.96. By this replacement, the conformational change caused an alteration in the ligand binding. The MolDock, PLANTS and LGA scores of ASP 137 ILE mutant were 
Torktaz I et al.

Table 1. Change in thermal stability and ligand binding of G. stearothermophilus amylase upon point mutation.

\begin{tabular}{|c|c|c|c|c|c|c|}
\hline $\begin{array}{l}\text { Amino acid } \\
\text { position }\end{array}$ & Native type & Mutant type & DDGP & Moldock score & PLANTS score & $\begin{array}{c}\text { Lamarkian Genetics } \\
\text { Algorithm }\end{array}$ \\
\hline Native & - & - & - & -152.245 & -76.246 & -6.3 \\
\hline 85 & A & $\mathrm{F}$ & -0.76 & -139.758 & -93.2712 & -6.3 \\
\hline 85 & A & I & -0.77 & -151.105 & -81.9656 & -6.3 \\
\hline 85 & A & W & -0.78 & -152.212 & -106.933 & -6.7 \\
\hline 85 & A & $\mathrm{Y}$ & -0.79 & -153.505 & -90.3072 & -6.8 \\
\hline 132 & M & I & -0.80 & -146.922 & -88.4827 & -6.2 \\
\hline 133 & $\mathrm{~K}$ & $\mathrm{~F}$ & -0.81 & -135.8444 & -78.9804 & -6.2 \\
\hline 133 & K & I & -0.82 & -153.539 & -103.5760 & -6.3 \\
\hline 133 & $\mathrm{~K}$ & $\mathrm{~L}$ & -0.83 & -146.554 & -90.9330 & -6.3 \\
\hline 133 & K & $\mathrm{V}$ & -0.84 & -153.516 & -95.6411 & -6.4 \\
\hline 133 & K & $\mathrm{Y}$ & -0.86 & -137.488 & -101.4340 & -6.4 \\
\hline 137 & $\mathrm{D}$ & $\mathrm{A}$ & -0.87 & -153.604 & -92.6331 & -6.4 \\
\hline 137 & $\mathrm{D}$ & $\mathrm{C}$ & -0.88 & -152.451 & -99.8759 & -6.2 \\
\hline 137 & $\mathrm{D}$ & $\mathrm{F}$ & -0.89 & -153.818 & -101.4110 & -6.4 \\
\hline 137 & $\mathrm{D}$ & $\mathrm{H}$ & -0.90 & -152.167 & -88.3625 & -6.3 \\
\hline 137 & $\mathrm{D}$ & I & -0.91 & -157.951 & -102.654 & -6.8 \\
\hline 137 & $\mathrm{D}$ & $\mathrm{L}$ & -0.92 & -153.555 & -85.5830 & -6.3 \\
\hline 137 & $\mathrm{D}$ & M & -0.93 & -136.168 & -92.6274 & -6.4 \\
\hline 137 & $\mathrm{D}$ & $\mathrm{R}$ & -0.94 & -153.830 & -99.0229 & -6.8 \\
\hline 137 & $\mathrm{D}$ & $\mathrm{T}$ & -0.96 & -154.935 & -89.2190 & -6.8 \\
\hline 137 & $\mathrm{D}$ & V & -0.97 & -155.478 & -102.1000 & -6.8 \\
\hline 137 & $\mathrm{D}$ & W & -0.98 & -152.127 & -90.4093 & -6.8 \\
\hline 137 & $\mathrm{D}$ & $\mathrm{Y}$ & -0.99 & -152.597 & -89.8772 & -6.8 \\
\hline 210 & $\mathrm{D}$ & $\mathrm{F}$ & -0.100 & -153.224 & -91.3364 & -6.0 \\
\hline 210 & $\mathrm{D}$ & $\mathrm{Y}$ & -0.102 & -147.386 & -90.8888 & -6.4 \\
\hline 211 & G & $\mathrm{C}$ & -0.103 & -152.486 & -90.0812 & -6.8 \\
\hline 211 & G & $\mathrm{F}$ & -0.104 & -142.984 & -92.6454 & -6.2 \\
\hline 211 & G & $\mathrm{H}$ & -0.105 & -148.336 & -101.7490 & -6.3 \\
\hline 211 & G & I & -0.106 & -143.028 & -101.4340 & -6.4 \\
\hline 211 & G & $\mathrm{L}$ & -0.107 & -153.786 & -88.3664 & -6.8 \\
\hline 211 & G & M & -0.108 & -153.636 & -100.7160 & -6.8 \\
\hline 211 & G & V & -0.109 & -141.354 & -101.7360 & -6.7 \\
\hline 211 & G & W & -0.110 & -145.346 & -88.9981 & -6.8 \\
\hline 211 & G & $\mathrm{Y}$ & -0.111 & -150.071 & -89.9360 & -6.8 \\
\hline 213 & $\mathrm{R}$ & $\mathrm{F}$ & -0.112 & -146.917 & -90.4186 & -6.9 \\
\hline 213 & $\mathrm{R}$ & I & -0.113 & -152.729 & -99.8250 & -6.8 \\
\hline 213 & $\mathrm{R}$ & W & -0.114 & -151.028 & -93.0541 & -6.4 \\
\hline 213 & $\mathrm{R}$ & $\mathrm{Y}$ & -0.115 & -153.647 & -100.2490 & -6.7 \\
\hline 215 & $\mathrm{D}$ & $\mathrm{F}$ & -0.116 & -146.689 & -95.7128 & -6.6 \\
\hline 215 & $\mathrm{D}$ & $\mathrm{H}$ & -0.117 & -153.274 & -91.0516 & -6.4 \\
\hline 215 & $\mathrm{D}$ & W & -0.118 & -153.461 & -89.7064 & -6.8 \\
\hline 215 & $\mathrm{D}$ & $\mathrm{Y}$ & -0.119 & -144.524 & -84.1400 & -6.8 \\
\hline 243 & G & $\mathrm{C}$ & -0.120 & -156.164 & -95.5001 & -6.4 \\
\hline 243 & G & F & -0.121 & -153.477 & -96.2011 & -6.8 \\
\hline 243 & G & I & -0.122 & -153.616 & -98.9821 & -6.7 \\
\hline 243 & G & $\mathrm{L}$ & -0.123 & -151.044 & -96.2164 & -6.7 \\
\hline 243 & G & M & -0.124 & -148.216 & -92.6612 & -6.8 \\
\hline 243 & G & V & -0.125 & -154.163 & -116.0010 & -6.8 \\
\hline 243 & G & W & -0.126 & -149.967 & -104.7340 & -6.7 \\
\hline 243 & G & Y & -0.127 & -156.385 & -96.4777 & -6.1 \\
\hline 244 & $\mathrm{E}$ & I & -0.131 & -157.114 & -109.4730 & -6.9 \\
\hline 244 & $\mathrm{E}$ & $\mathrm{L}$ & -0.132 & -144.578 & -99.4078 & -6.5 \\
\hline 244 & E & M & -0.133 & -149.747 & -91.9512 & -6.6 \\
\hline 244 & E & $\mathrm{S}$ & -0.136 & -151.154 & -103.4900 & -6.8 \\
\hline 244 & E & $\mathrm{T}$ & -0.137 & -153.599 & -102.9570 & -6.8 \\
\hline 244 & E & V & -0.138 & -156.504 & -112.5900 & -6.8 \\
\hline 244 & $\mathrm{E}$ & $\mathrm{Y}$ & -0.140 & -156.504 & -98.3125 & -6.9 \\
\hline
\end{tabular}




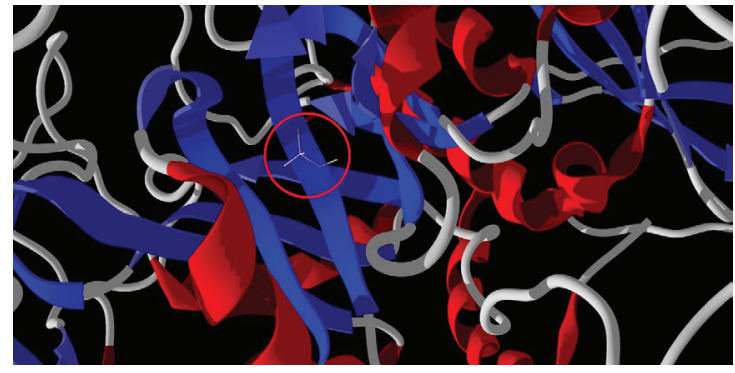

Figure 3. The position of Gly 243 in the middle of a beta sheet in the core of G. stearothermophilus alpha amylase.

$-157.951,-102.654$, and -6.8 , respectively. These scores indicate that there are considerable differences between the native enzyme and ASP 137 ILE mutant in the ligand binding avidity. In the other words, the affinity of this mutant enzyme is theoretically increased toward amylose. Likewise, mutating aspartic acid 137 to valine will result in a stronger beta sheet's hydrophobic core and an alteration in the structure that changes the ligand binding affinity to -155.478 for MolDock, -102.1000 , and 6.8 for the PLANTS and LGA, respectively. Interestingly by replacing aspartic acid 137 to valine, the thermal stability of the enzyme increased by 0.97 . In position 243 there is a Glycine and is located in the middle of the beta strand in the core of the protein (Fig. 3 ). Glycine naturally tends to make turns and loops. So, its existence in the middle of a beta strand leads to the instability. According to Table 1, replacing Glycine with various amino acids increases the thermal stability, but just in one replacement G243V results in an increased ligand binding potential. The MolDock, PLANTS, and LGA scores for this mutation were -154.163, -116.001 , and -6.8 , respectively. Following the amino acid sequence of the enzyme, right after Gly there is a Glutamic acid at position 244. The $\mathrm{pK}_{\mathrm{a}}$ of the side chain of the Glutamic acid is 4.25 while its $\mathrm{pI}$ is 3.0. So, in the neutral $\mathrm{pH}$, it is positively charged, and probably, it creats a local charge imbalance in the core of the protein.

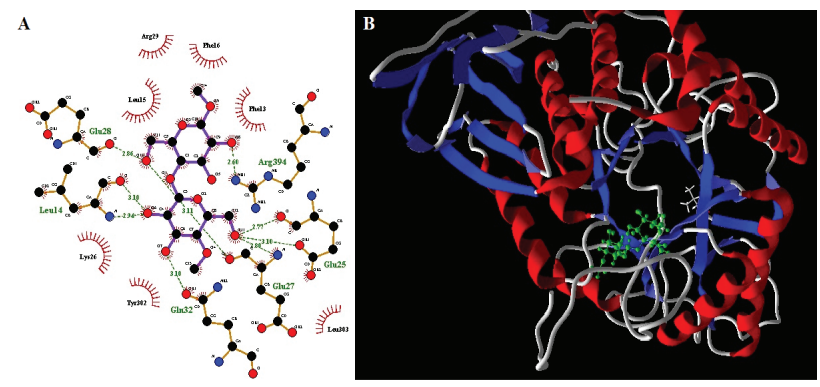

Figure 4. (A) The ligand map of the theoretical recombinant model of Asp 137 Val. (B) The docking view of the Asp 137 Val. The replaced amino acid is depicted in the nearby of the binding site.
As described in the Table 1, replacing GLU 244 with the negatively charged amino acids or hydrophobic ones renders the protein to a more stable enzyme. Replacing GLU 244 with the isoleucine and tyrosine alters the structure in a manner that the consequence would be an increased ligand binding (Figs. 5, 6, and 7). The results of obtained computational data in the $\alpha$-amylase from Geobacillus stearothermophilus is compairable with the serults of thermal stability enhancement by single point substitution on Cel5E from Clostridium

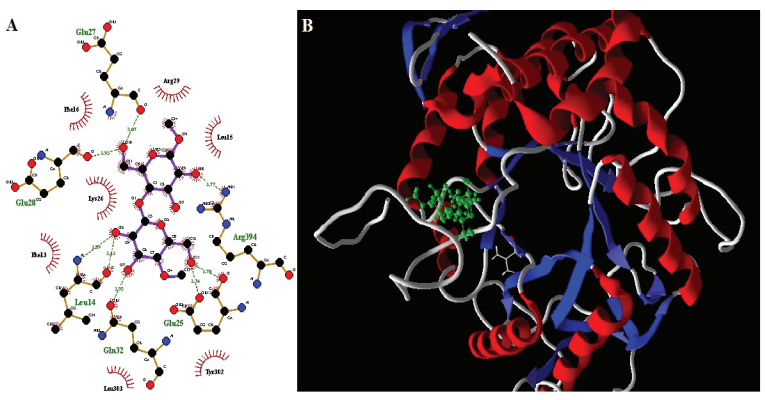

Figure 5. (A) The ligand map of $243 \mathrm{GV}$. (B) The docking view of $243 \mathrm{GV}$ which indicates that this replacement directly affects the coordinate of the binding site.

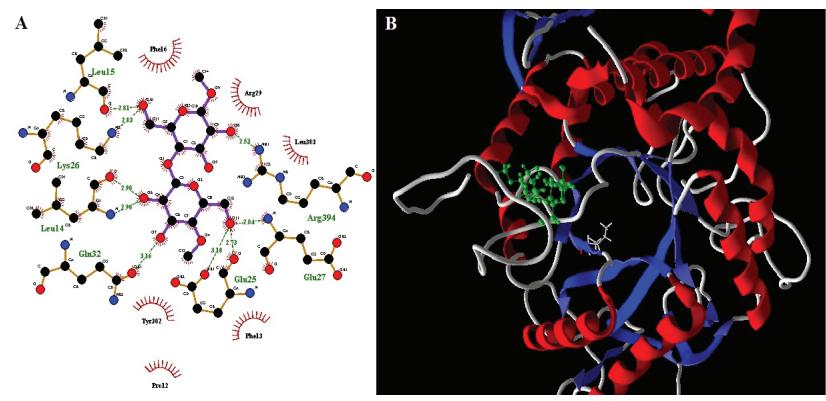

Figure 6. (A) The ligand map of 244 EI mutant. (B) The docking view of 244EI indicates the position of the replaced residue near to the active site.

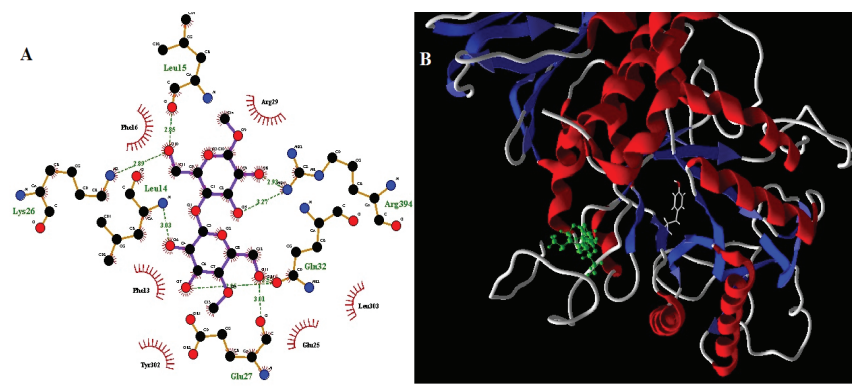

Figure 7. (A) The ligand map of 244 EY mutant. (B) The docking view of the $244 \mathrm{EY}$ indicates that the $\mathrm{R}$ group of the replaced residue does not directly in contact with the active site, but the substitution has a direct effect on the overall coordinate of the local backbone and by structural change increases the binding avidity. 
thermocellum. In the positions of Asn 207 several point mutations could increase the enzyme stability and activity. Asn207Arg could change the $\Delta \Delta \mathrm{G}$ by 1.27 and increase the ligand biding affinity from 64.9 (MolDock scale) to 102.6. Moreover, Asn207Lys also increased the thermal stability by $\Delta \Delta \mathrm{G}=1.76$ and the ligand biding affinity improved by 43.7 in MolDock scale. This in silico observation is due to: (i): omitting Asn which is a residue with deamination potential that mekae its local area unsbale. (ii): new hydrogen bond formation by side chain of Lys or Arg as the substituent. This observation is compairable with a study by Micheus et al., which revealed that Gln264Ser/Asn265Tyr mutations that omits deamination potential, lead to more thermal stability in the amylase from Bacillus licheniformis (25). In another study, Asn188Thr mutation on alpha amylase from Bacillus licheniformis could increase the thermal stability of protein by making salt bridge bond with Thr263 (26). Our observations in this study suggested that best mutations for making G. stearothermophilus amylase more thermo tolerant with more ligand binding avidity are ASP137VAL, GLY243VAL, GLU244ILE and GLU244TYR, acordingly. By adding VAL and ILE as substituents, the local hydrophobicity of the protein has increased. It lead to expanding hydrophobic interactions in the positions of 137, 243 and 244. Therefore, the thermal stability of the enzyme should be increased with the aim of extra intera molecular hydrophobic interactions. This observation is compairable with a study on maltohexaose-forming $\alpha$-amylase from Bacillus stearothermophilus, which revealed that Asn193Phe mutation in combination with Ser242Ala could increase the half life of the enzyme 26fold at $95{ }^{\circ} \mathrm{C}(27)$. In another study, hyperthermostable mutants of Bacillus licheniformis alpha-amylase were generated by replacing His 133 and Ala209 to Ile and Val. New mutant enzyme could retian its half life more 3 folds at 80 degrees C (28). Moreover, it has been indicated that stabilizing hydrophobic substitutions compensated hydrogen bonds of His and increased hydrophobic side-chain interactions within the betasheet where residue 133 is located (29).

\section{Conclusion}

The major conclusion of this study is that a series of thermal stability increasing point mutations were excuted around the active site of the enzyme, the binding affinity was measured, and finally a list of mutations that have increased the thermal stability as well as catalytic activity were proposed. The results of this in silico study could be used for further downstream laboratory experiments toward achieving an enzyme with both higher thermally stable and catalytically active enzymes.

\section{Acknowledgment}

This research is supported by the National Institute of Genetic Engineering and Biotechnology (NIGEB), Iran.

\section{Spplementary Data}

This paper includes a supplementary file containing details of delta $\mathrm{G}$ calculation and molecular docking process which is appeared on the web site of the IJB journal (http://www.ijbiotech.com).

\section{References}

1. Demirjian DC, Moris-Varas F, Cassidy CS. Enzymes from Extremophiles. Curr Opin Chem Biol. 2001;5(2):144-51. doi: 10.1016/S1367-5931(00)00183-6.

2. Zeikus JG, Vieille C, Savchenko A. Thermozymes: Biotechnology and Structure-Function Relationships. Extremophiles. 1998;2(3):179-83. doi: 10.1007/s007920050058.

3. Potapov V, Cohen M, Schreiber G. Assessing Computational Methods for Predicting Protein Stability upon Mutation: Good on Average but not in the Details. Protein Eng Des Sel. 2009;22(9):553-60. doi: 10.1093/protein/gzp030.

4. Turner P, Mamo G, Karlsson EN. Potential and Utilization of Thermophiles and Thermostable Enzymes in Biorefining. Microb Cell Fact. 2007;6:9. doi: 10.1186/1475-2859-6-9.

5. Kristjansson JK. Thermophilic Organisms as Sources of Thermostable Enzymes. Trends Biotechnol. 1989;7(12):349-53. doi: 10.1016/0167-7799(89)90035-8.

6. MacGregor EA, Janecek S, Svensson B. Relationship of Sequence and Structure to Specificity in the Apha-amylase Family of Enzymes. Biochim Biophys Acta. 2001;1546(1):1-20. doi: 10.1016/S0167-4838(00)00302-2.

7. de Souza PM, de Oliveira Magalhaes P. Application of Microbial Alpha-amylase in Industry - A Review. Braz J Microbiol. 2010;41(4):850-61. doi: 10.1590/S1517-83822010000400004.

8. Rajagopalan G, Krishnan C. Alpha-amylase Production from Catabolite Derepressed Bacillus subtilis KCC103 Utilizing Sugarcane Bagasse Hydrolysate. Bioresour Technol. 2008;99(8):3044-50. doi: 10.1016/j.biortech.2007.06.001

9. Kagawa M, Fujimoto Z, Momma M, Takase K, Mizuno H. Crystal Structure of Bacillus subtilis Alpha-amylase in Complex with Acarbose. \J Bacteriol. 2003;185(23):6981-4. doi: 10.1128/ JB.185.23.6981-6984.2003.

10. Stamford TL, Stamford NP, Coelho LC, Araujo JM. Production and Characterization of a Thermostable Alpha-amylase from Nocardiopsis sp. Endophyte of Yam bean. Bioresour Technol. 2001;76(2):137-41. doi: 10.1016/S0960-8524(00)00089-4.

11. Zhou T, Dong Z, Setlow P, Li YQ. Kinetics of Germination of Individual Spores of Geobacillus stearothermophilus as Measured by Raman Spectroscopy and Differential Interference Contrast Microscopy. PloS one. 2013;8(9):e74987. doi: 10.1371/ journal.pone.0074987.

12. Tomazic SJ, Klibanov AM. Mechanisms of Irreversible Thermal Inactivation of Bacillus Alpha-amylases. J Biol Chem. 1988;263(7):3086-91.

13. Ben Ali M, Khemakhem B, Robert X, Haser R, Bejar S. Thermostability Enhancement and Change in Starch Hydrolysis Profile of the Maltohexaose-Forming Amylase of Bacillus stearothermophilus US100 Strain. Biochem J. 2006;394(Pt 1):51- 
6. doi: 10.1042/BJ20050726.

14. Vieille C, Zeikus GJ. Hyperthermophilic Enzymes: Sources, Uses, and Molecular Mechanisms for Thermostability. MMBR. 2001;65(1):1-43. doi: 10.1128/MMBR.65.1.1-43.2001

15. Khan S, Vihinen M. Performance of Protein Stability Predictors. Hum Mutat. 2010;31(6):675-84. doi:10.1002/humu. 21242.

16. Dehouck Y, Grosfils A, Folch B, Gilis D, Bogaerts P, Rooman M. Fast and Accurate Predictions of Protein Stability Changes upon Mutations Using Statistical Potentials and Neural Networks: PoPMuSiC-2.0. Bioinformatics (Oxford, England). 2009;25(19):2537-43. doi: 10.1093/bioinformatics/btp445.

17. Razvi A, Scholtz JM. Lessons in Stability from Thermophilic Proteins. Protein Sci. 2006;15(7):1569-78. doi:10.1110/ ps.062130306.

18. Sanchez-Ruiz JM, Makhatadze GI. To Charge or not to Charge. Trends Biotechnol. 2001;19(4):132-5. doi: 10.1016/S01677799(00)01548-1.

19. Karplus K. SAM-T08, HMM-based Protein Structure Prediction. Nucleic Acids Res. 2009;37(Web Server issue):W492-7. doi: 10.1093/nar/gkp403

20. Benkert P, Tosatto SC, Schomburg D. QMEAN: AComprehensive Scoring Function for Model Quality Assessment. Proteins. 2008;71(1):261-77. doi: 10.1002/prot.21715.

21. Gilis D, Rooman M. PoPMuSiC, An Algorithm for Predicting Protein Mutant Stability Changes: Application to Prion Proteins. Protein Eng. 2000;13(12):849-56. doi: 10.1093/ protein/13.12.849.

22. Thomsen R, Christensen MH. MolDock: A New Technique for High-accuracy Molecular Docking. $J$ Med Chem. 2006;49(11):3315-21. doi: 10.1021/jm051197e.
23. Vahed M, Motalebi E, Rigi G, Akbari Noghabi K. Soudi MR, Sadeghi M, Ahmadian G. Improving the Chitinolytic Activity of Bacillus pumilus SG2 by Random Mutagenesis. J Microbiol Biotechnol 2013;23:1519-1528. doi: 10.4014/jmb.1301.01048.

24. Herav K, Rigi M, Rezaei G. Arjoman M, Rostami A, Ahmadian G. An Alternative Bacterial Expression System Using Bacillus pumilus SG2 Chitinase Promoter. Iran J Biotechnol. 2016; 13 (4), 17-24. doi:10.15171/ijb.1175.

25. Machius M, Declerck N, Huber R, Wiegand G. Kinetic stabilization of Bacillus licheniformis alpha-amylase through introduction of hydrophobic residues at the surface. J Biol Chem. 2003; 278 (13):11546-53. doi: 10.1074/jbc.M212618200.

26. Li Z, Duan X, Chen S, Wu J. Improving the reversibility of thermal denaturation and catalytic efficiency of Bacillus licheniformis alpha-amylase through stabilizing a long loop in domain B. PLoS One. 2017; 12 (3): e0173187. doi:10.1371/journal.pone.0173187.

27. Li Z, Duan X, Wu J. Improving the thermostability and enhancing the $\mathrm{Ca}(2+)$ binding of the maltohexaose-forming alpha-amylase from Bacillus stearothermophilus. J Biotechnol. 2016; 222: 6572. doi:10.1016/j.jbiotec.2016.02.013.

28. Declerck N, Joyet P, Trosset JY, Garnier J, Gaillardin C. Hyperthermostable mutants of Bacillus licheniformis alphaamylase: multiple amino acid replacements and molecular modelling. Protein Eng. 1995; 8(10): 1029-37. doi:10.1093/ proeng/gzg032.

29. Declerck N, Machius M, Chambert R, Wiegand G, Huber R, Gaillardin C. Hyperthermostable mutants of Bacillus licheniformis alpha-amylase: thermodynamic studies and structural interpretation. Protein Eng. 1997; 10(5): 541-9. doi:10.1093/protein/10.5.541. 